Q

\title{
Sustaining energy systems using metal oxide composites as photocatalysts
}

Mir Sayed Shah Danish¹, Tomonobu Senjyu ${ }^{2 *}$, Abdul Matin Ibrahimi², Arnab Bhattacharya ${ }^{3}$, Zahra Nazari ${ }^{4}$, Sayed Mir Shah Danish ${ }^{5}$ and Mikaeel Ahmadi ${ }^{2}$

1Strategic Research Project Center, University of the Ryukyus, 1 Senbaru 9030213, Japan

2Department of Electrical and Electronics Engineering, Faculty of Engineering, University of the Ryukyus, Okinawa 903-0213, Japan

${ }^{3}$ Department of Academic Affairs, Research and Education Promotion Association (REPA), Okinawa 900-0015, Japan

${ }^{4}$ Department of Computer Science, Kabul Polytechnic University, Kabul 1006, Afghanistan

${ }^{5}$ Department of Electrical Engineering, Technical Teachers Training Academy (TTTA), Chihl Sutton, Kabul, Afghanistan

Article

Open Access

Published

Keywords

- Metal-oxide

- Metal oxide-MOFs composition

- Metal oxide-carbon material composition

- Photocatalysts

- Storage systems materials

- Sensors

- Carbon dioxide reduction

- Environmental sustainability
ABSTRACT

Among the various types of metal organic frameworks (MOFs), the metal-oxide-based ones fulfill all the essential criteria such as strong bonding, organic linking units, and highly crystalline nature, properties required to be effective photocatalysts to serve environmental remediation. Moreover, the even spread of active sites and semiconductor properties make the MOFs ideal for absorbing irradiation from UV as well as visible light sources. Metal oxide composites with carbon based materials, especially, show high photocatalytic activity toward the degradation of organic dyes. Considering the relatively low cost of metal oxide semiconductors compared to pure metallic nanoparticles, metal oxide composites can provide a great alternative as photocatalysts especially considering the adjustable bandgaps and synergistic effects. Therefore, the metal oxide application as the photocatalysts in industry and technology in terms of techno-economic advantage is attracted. In this study, energy sustainability and solving carbon-related issues through metal oxide-based materials are discussed. This study aims to review metal oxide composites including metal oxide-MOFs and metal oxide-carbon material compositions as photocatalysts, application, merits in environmental and energy systems performances, and its contribution as an influential factor for sustainable development.

Received: July 20, 2021; Revised: August 07, 2021; Accepted: August 12, 2021; Published: August 20, 2021

(C2021 REPA. All rights reserved.

\section{Introduction}

In a novel approach, MIL-125- $\mathrm{NH}_{2}$ was doped with $\mathrm{Ag}_{3} \mathrm{VO}_{4}$ and $\mathrm{Ag}_{2} \mathrm{WO}_{4} \mathrm{NPs}$, which reduced the bandgap of the MIL from $2.65 \mathrm{eV}$ to $2.56 \mathrm{eV}$ and $2.27 \mathrm{eV}$, respectively. The organic dyes $\mathrm{MB}$ and $\mathrm{RhB}$ were degraded under visible light irradiation was more than the MIL by about 2.1 to 4.9 times [1]. Incorporation of $\mathrm{Fe}_{3} \mathrm{O}_{4} \mathrm{NPs}$ into the MIL-100 by a solvothermal process afforded core/shell encapsulation. The as-prepared $\mathrm{Fe}_{3} \mathrm{O}_{4} @ M I L-100$, due to its magnetic property, permitted easy recovery of the photocatalysts from the reaction medium in the breakdown of MB dye [2]. Another semiconductor metal oxide $\mathrm{ZnO}$, has been under research for potential photocatalytic applications as part of MOF composites. ZnO@MOF-46 and ZnO@ZIF-8 have been investigated as potential candidates for wastewater treatment to remove $\mathrm{MB}$ and $\mathrm{Cr}(\mathrm{VI})$, respectively. In the case of ZnO@MOF-46 photocatalyst, the role of ${ }^{\circ} \mathrm{OH}$ was prominent in accelerating the decolorization of MB. The dye was degraded up to $81 \%$ in $3 \mathrm{~h}$ under UV irradiation which was a significant improvement over the $41.5 \%$ and $51 \%$ in the presence of individual $\mathrm{ZnO}$ and MOF-46, respectively [3]. The formation of ZnO@ZIF-8 core-shell heterostructures depended on the concentration of 2-methylimidazole during its synthesis using $\mathrm{ZnO}$ colloidal spheres as templates. Selective reduction of $\mathrm{Cr}(\mathrm{VI})$ was observed due to the selective adsorption and permeation induced by ZIF-8 shell. On the other hand, MB molecules could neither be adsorbed onto its surface nor be permeated into the MOF, which led to the selectivity of $\mathrm{Cr}(\mathrm{VI})$ over MB [4]. Fast degradation of RhB was reported by Mahmoodi et al. who utilized a two-step process to obtain the photocatalyst Ag/AgCl@CFNMT. The synthesis involved initial magnetization of the MOF NH 2 -MIL-125(Ti) (NMT) by $\mathrm{CoFe}_{2} \mathrm{O}_{4}$ followed by an in situ deposition/photo-reduction technique to assemble $\mathrm{Ag} / \mathrm{AgCl}$ onto the surface of $\mathrm{CoFe}_{2} \mathrm{O}_{4}$ /NMT (CFNMT). The synergistic effect observed between the $\mathrm{Ag} / \mathrm{AgCl}$ and CFNMT regions propelled a superfast degradation under LED visible light. Photodegradation ability of $89 \%$ was achievable with this nanocomposite even after seven cycles, and its easy separation from the reaction medium was due to the magnetic properties [5].

Considering the relatively low cost of metal oxide semiconductors compared to pure metallic nanoparticles, the composites 'MO@MOF' can provide a great alternative as photocatalysts especially considering the adjustable bandgaps and synergistic effects [6]. The addition of dye sensitizers to this combination can further enhance the photocatalytic activity of the metal oxide semiconductor. In this context, Xie et al. [7] reported the introduction of MOF as a sensitizer for anatase $\mathrm{TiO}_{2}$. ADA-Cd crystals, when mixed physically with $\mathrm{TiO} 2$ at specific proportion, yielded $\mathrm{ADA}-\mathrm{Cd} / \mathrm{TiO}_{2}$, which was efficient for the task of 
degrading organic dyes under irradiation of visible light via photocatalysis. The bandgap of $\mathrm{ADA}-\mathrm{Cd} / \mathrm{TiO}_{2}$ being $3.14 \mathrm{eV}$, provided an excellent example as to how the fabrication of metal oxide-MOFs can tune the absorption of light. This modified bandgap provided the perfect platform for the photocatalytic activity of $\mathrm{ADA}-\mathrm{Cd} / \mathrm{TiO}_{2}$ toward organic dyes under visible light, which could not be matched by either $\mathrm{TiO}_{2}(3.20 \mathrm{eV})$ or $\mathrm{ADA}-\mathrm{Cd}(3.04 \mathrm{eV})$ alone. This observation confirmed the synergistic mechanism between the $\mathrm{TiO}_{2}$ and the coordinated ADA-Cd MOFs. Electrons present in the conduction band of $\mathrm{TiO}_{2}$ were found to take up $\mathrm{O}_{2}$ from the atmosphere and oxidize it into the form of key oxidative species. The holes present in the valence band of the MOF, in tandem with ${ }^{\circ} \mathrm{OH}$ resulting from another mechanism, assisted the photodegradation [7]. Selective removal of cationic dyes was achieved by $\mathrm{Fe}_{3} \mathrm{O}_{4} @ H P U-9$ composites having core-shell structures. The removal of R6G could be performed continuously for several cycles without the need for centrifugal separation of the catalyst [8]. A simple technique of fabricating $\mathrm{Fe}_{3} \mathrm{O}_{4} / \mathrm{Cu}_{3}(\mathrm{BTC})_{2}$ by controlling the rate of ligand addition was demonstrated by Zhao et al. The synthesized metal oxide-MOF composite was tested for its catalytic activity in removal of MB from water in the $\mathrm{pH}$ range of 2-11. Due to the development of negative charge on the adsorbent at higher $\mathrm{pH}$ values, the electrostatic interaction between these and the cationic dye was found to be higher but not the major cause of adsorption. Rather, the $\pi-\pi$ stacking and hydrophobic interactions between the $\mathrm{Fe}_{3} \mathrm{O}_{4} / \mathrm{Cu}_{3}(\mathrm{BTC})_{2}$ surface and MB resulted in the adsorption that followed the pseudo-first order kinetic model. This system also provided a low cost alternative to other adsorbents [9]. Engineering metal-Organic frameworks (MOFs) for controlled delivery of physiological gaseous transmitters discussed in [10], tried to propose an emerging field to develop signaling molecule-releasing MOFs with practical applications.

Poly aromatic hydrocarbons (PAHs) are one of the major environmental pollutants arising out of rapid industrialization and their extraction mechanisms remain under intense research. MOFs have also been researched under this field and very recently, Li et al. have synthesized $\mathrm{Fe}_{3} \mathrm{O}_{4} @$ MIL-101 nanocomposites made up of $\mathrm{Fe}_{3} \mathrm{O}_{4}$ core and MIL-101 (Cr) MOF shell as a viable material for negligible-depletion magnetic solid-phase extraction (ndMSPE) of PAHs dissolved freely in aquatic bodies. $\pi-\pi$ interactions, $\pi$-complexation and hydrophobic interactions between the nanocomposites and PAHs resulted in remarkable lowering the time for extraction equilibrium to less than $60 \mathrm{~min}$. Competitive substances like DOM and DOM-associated PAHs are not adsorbed while the PAHs can be detected up to $0.08-0.82 \mathrm{ng} \mathrm{L}^{-1}$ and adsorbed due to the porous structure of the nanocomposites [11].

Soil contaminant pesticides such as organophosphorus glyphosate $\left(\mathrm{C}_{3} \mathrm{H}_{8} \mathrm{NO}_{5} \mathrm{P}\right)$ are extremely harmful to aquatic plants and microbes and hence their detection is of immense value for environmental remediation. In this context, the work of Yang et al. provides a Zr-MOF based composite that can detect glyphosate at concentrations up to $0.093 \mathrm{mg} / \mathrm{L}$. The $\mathrm{Zr}-\mathrm{OH}$ groups were found to act as the functional groups in the composite $\mathrm{Fe}_{3} \mathrm{O}_{4} @ \mathrm{SiO}_{2} @ \mathrm{UiO}-67$ owing to their high affinity toward the phosphate groups. The electron transfer between the $\mathrm{Fe}_{3} \mathrm{O}_{4}$ and UiO-67 components was hindered to a large extent by the intervening $\mathrm{SiO}_{2}$. As a consequence, the detection was possible at such a low limit of glyphosate concentration. The binding of the adsorbent with the phosphate groups was indicated by a distinct change in the fluorescence intensity at $315 \mathrm{~nm}$ [12]. Metal-oxide based materials and photocatalysts were also applied for carbon dioxide reduction through systematic steps as [13]:

- Adsorption of reductant and $\mathrm{CO}_{2}$ onto the metal oxide catalyst surface;

- Adsorption of sunlight on the perovskite leading to electron-hole separation;

- Transfer of photogenerated charges to the surface and reaction with adsorbed $\mathrm{CO}_{2}$ and water;

- Desorption of products.

Danish et al. introduced semiconductor metal oxides such as $\mathrm{TiO} 2, \mathrm{ZnO}, \mathrm{WO} 3, \mathrm{BiVO} 4$ and $\mathrm{Fe} 2 \mathrm{O} 3$ for photoelectrochemical water splitting and photoelec-trochemical (PEC) water splitting as solar-driven water splitting methods to produce hydrogen gas in the context of environmentally friendly and sustainable energy production [14]. Antibiotics being highly toxic toward microbial life can cause substantial damage to environment. As a consequence, the removal of antibiotics is an area of focused research. Highly crystalline $1 \mathrm{D}$ MIL- $100(\mathrm{Fe}) / \mathrm{TiO}_{2}$ nanoarrays were synthesized by Xiang He et al. for tetracycline removal at an efficiency of $90.79 \%$. The degradation carried out in quartz cuvette under ambient conditions displayed good recyclability of the catalyst, which was recorded to be twice as high as pure titanium oxide, even after five cycles [15]. Ciprofloxacin is a common antibiotic that is among the most persistent pharmaceuticals present in wastewaters and needs efficient removal. In view of this, Moradi et al. developed magnetic metal-organic framework sorbents $\mathrm{Fe}_{3} \mathrm{O}_{4} @$ MIL-100(Fe) and $\mathrm{Fe}_{3} \mathrm{O}_{4} @ M O F-$ $235(\mathrm{Fe})$ for CIP removal which that occurred in a spontaneous and endothermic manner. While $\mathrm{Fe}_{3} \mathrm{O}_{4} @ \mathrm{MIL}-$ $100(\mathrm{Fe})$ displayed both chemisorption and physisorption, the latter only showed physisorption. Phase separation was also easily achieved on using the $\mathrm{Fe}_{3} \mathrm{O}_{4} @ \mathrm{MIL}-100(\mathrm{Fe})$ composite [16].

Heavy metal removal using 'MO@MOF' composites was demonstrated by employing the magnetic Fe304@UiO-66 produced by the epitaxial method. A lot of micropores and a large surface area attributed to the composites' coreshell structure allowed for multi-layer heterogeneous adsorption of As (V) up to $73.2 \mathrm{mg}$ per gram of the adsorbent. The inclusion of the ferrosoferric oxide provided good 
thermal stability in pseudo-second-order kinetics and easy separation from the medium due to its magnetic nature [17]. Used as a disinfectant widely, and especially during the large-scale breakout of infectious diseases, Hypochlorous acid ( $\mathrm{HClO}$ ) finds its way into the environment, leading to severe ramifications. Therefore, the detection of HClO levels is an important step toward limiting its usage. Ma et al. developed $\mathrm{ZIF}-8 / \mathrm{RhB}-\mathrm{CDs} / \mathrm{Fe}_{3} \mathrm{O}_{4}$ as a ratiometric and magnetic probe for fluorimetric detection of HClO. While Rhodamine B carbon dots and ferrosoferric oxide are the guest particles in this system, the MOF ZIF-8 acts as the platform to house these. The role of the MOF also extends to the adsorption of the analyte HClO. Extreme fluorescence quenching at $580 \mathrm{~nm}$, almost thrice as much as the RhB-CDs probe, confirmed the detection even at a LOD of $6.7 \mu \mathrm{M}$. The role of $\mathrm{Fe}_{3} \mathrm{O}_{4}$ was to provide easy magnetic separation of the nanoprobes [18].

\section{Metal oxide-carbon material composites as photo- catalysts}

Balancing The rise of polymers, carbon fibers, graphene oxides, fullerenes, nanotubes, quantum dots and other carbon-based materials has opened up many research opportunities and their practical applications. Research on environmental remediation has also seen the use of such materials in conjugation with other materials through interface engineering, doping, surface functionalization, and coordination, for achieving effective results.

Metal oxide composites with carbon-based materials, especially, show high photocatalytic activity toward the degradation of organic dyes. In one such example, dispersion of Pt particles in the composite of ZnO/CFs. The Pt@ZnO NRs/CFs composites were prepared via a three-step route involving atomic layer deposition of $\mathrm{Pt}$, hydrothermal growth, and magnetron sputtering. Synergistic effect between the Pt NPs and the ZnO nanorods facilitated easy electron transfer at their interface and impeded the electron-hole recombination to a great extent. Therefore, the photocatalytic activity in the degradation of organic dyes increased tremendously, as shown in the case of methyl orange degradation. The hierarchical structure provided the necessary stability [19]. Bark derived from Konar tree was used to make activated carbon further used in preparing modified $\mathrm{ZnO}$ nanoplates. Under visible light irradiation, this $\mathrm{ZnO}$-AC composite showed great catalytic activity in removing CIF from aqueous solution [20]. In another instance, direct hydrothermal method was used to synthesize ZnO NPs decorated nitrogen-doped graphite carbon sheets from peach fruit juice. These ZnO@N-Cs were used to determine the effectiveness in MB removal and the results revealed these composites to be more than $95 \%$ efficient for the purpose. Use of natural bio-waste as the raw material for the synthesis, neutral aqueous solution for the degradation study and a degradation time of less than 60 minutes suggested these composites to be ideal for practical purposes [21].

Different ratios of $\mathrm{ZnO}$ nanorods and graphene oxide were used to prepare $\mathrm{ZnO}$-rGO NCs. Among these, the $\mathrm{ZnO}-5 \%$ rGO displayed very good photocatalytic activity for the degradation of RhB. It was six times more than pure $\mathrm{ZnO}$ NPs and two times more than $\mathrm{ZnO}-5 \% \mathrm{rGO}(\mathrm{H})$. In the degradation of phenol, activity enhancement was thrice that of $\mathrm{ZnO}-5 \% \mathrm{rGO}(\mathrm{H})$. The significant aspect of these NCs was that they could work in the photodegradation of both the positively charged MB and the negatively charged phenol species in aqueous medium. Higher surface area and a higher separation between the electron hole pairs result in the heightened activity in photodegradation. Different key radicals were formed during the degradation of phenol and MB, which suggested the versatility of the prepared NCs [22]. Hydrothermal and sol-gel methods were used by Jo et. al. to prepare a series of binary and ternary titania based nanocomposites. Homogeneous dispersion of $\mathrm{Co}_{3} \mathrm{O}_{4}$ over the surface of $\mathrm{TiO}_{2} \mathrm{NPs}$ resulted in remarkable increase in visible light driven photocatalysis. The degradation of oxytetracycline (OTC) and Congo red (CR) in aqueous medium was further improved by modifying this $\mathrm{TiO}_{2}-\mathrm{Co}_{3} \mathrm{O}_{4}$ nanocomposite with $\mathrm{GO}$-nanosheets in trace amount. The electron-hole recombination was effectively prevented by the addition of $\mathrm{GO}$, while the combination of $\mathrm{TiO}_{2}$ and $\mathrm{Co}_{3} \mathrm{O}_{4}$ modulated the bandgap (Figure 1) [23].

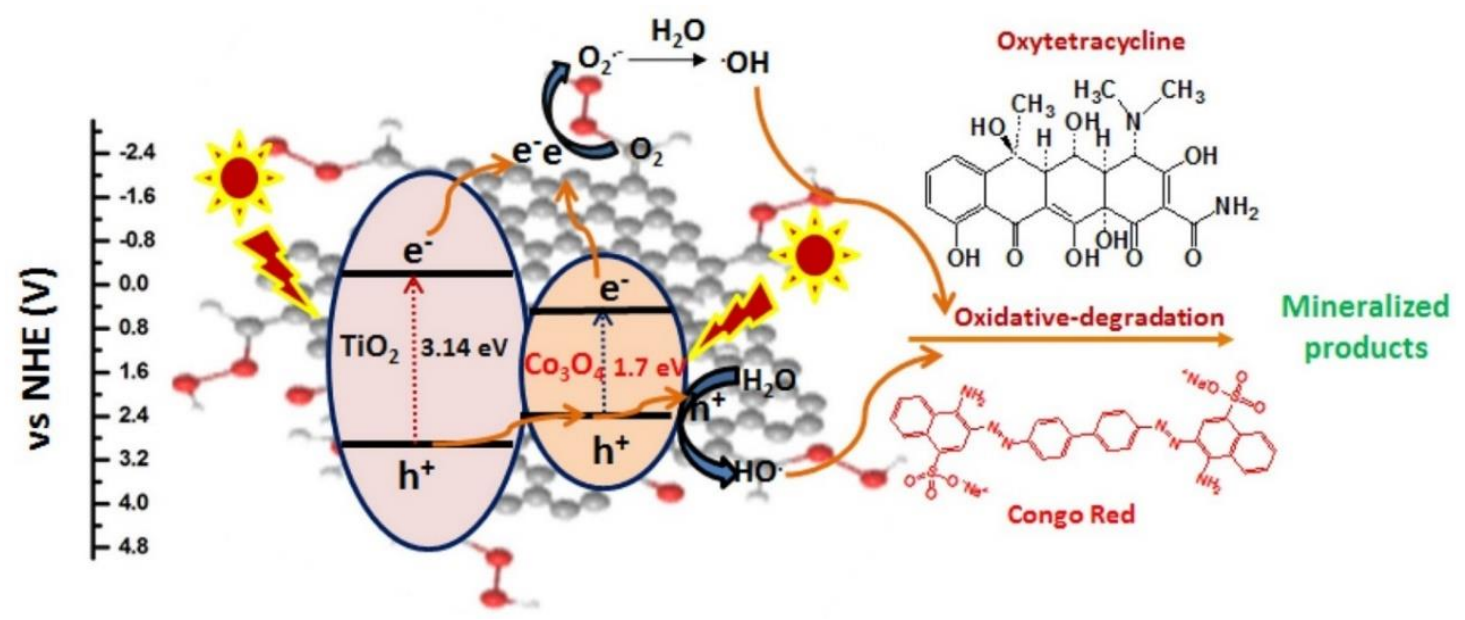

Figure 1. Photocatalysis mechanism of OTC and CR by ternary titania based nanocomposites [23]. 
A well-regulated hydrothermal synthesis was used to obtain the composites of $2 \mathrm{D}-\mathrm{WO}_{3}$ sheets and reduced GO. Under sunlight, these $\mathrm{rGO} / \mathrm{WO}_{3} \mathrm{NCs}$ displayed photodegradation of $\mathrm{MB}$ and $\mathrm{RhB}$, which was found to be better than that of pure $\mathrm{WO}_{3}$ [24]. Uniform dispersion of $\mathrm{WO}_{3}$ sticks inside the graphene sheets was achieved by hydrothermal process that resulted in the $\mathrm{WO}_{3} \mathrm{NR} / \mathrm{G}$ composites. The variant with $7 \% \mathrm{G}\left(\mathrm{WO}_{3} / \mathrm{G}-7\right)$ exhibited the most efficient photodegradation of $\mathrm{MB}$ under visible light irradiation. The reason for the same was considered to be the decrease in the bandgap energy in $\mathrm{WO}_{3}$ [25]. Taha et al. synthesized $\mathrm{WO}_{3}-\mathrm{CNF}$ NCs employing an electrospinning method along with carbonization. The incorporation of CNF into the metal oxide semiconductor provided a way to develop high-efficiency NCs that displayed photocatalytic properties in the degradation of MB, MO, and MG in aqueous medium [26]. The synthesis of composites containing carbon nanodots and $\mathrm{WO}_{3}$ via a hydrothermal process was reported by Song et al. Efficient light-harvesting and superior electron-hole separation, as a result of the synergistic effect between the metal oxide and the carbon dots, were the hallmark of this system which made the Cdots/ $\mathrm{WO}_{3}$ as efficient photocatalysts for the degradation of RhB under visible light irradiation [27]. Effective degradation of Indigo carmine (IC) and MB were realized under sunlight by the ultrasonically prepared $\mathrm{WO}_{3}-\mathrm{GO} \mathrm{NCs}$. The activity was better than the pure and, therefore, a significant achievement in the easy synthesis active photocatalyst composites of metal oxides and graphene [28]. Polyvinylidene fluoride and polyvinylpyrrolidone were used as the base material to support $\mathrm{TiO}_{2}$ NPs. Electrospinning technique was used to fabricate the PVDF-PVP base wherein the PVP could be dissolved to increase the total surface area and make way for the reactive species to access the $\mathrm{TiO}_{2}$ NPs. This arrangement provided both stability and enhancement of performance as demonstrated by the photo degradation of MB which worked with high efficiency even after 10 cycles [29].

Degradation of 2,4 dicholorphenol is extremely important since this contaminant causes liver and kidney related problems in addition to nervous system damage in humans. In this direction, the synthesis of $\mathrm{Fe}_{3} \mathrm{O}_{4} @ \mathrm{GO} @ \mathrm{MIL}-100$ (Fe) composite by Gong et al. is an interesting report. Degradation efficiency of $100 \%$ in 60 minutes in the presence of hydrogen peroxide was achieved owing to the fast transfer of photogenerated electrons between all the three components, i.e. $\mathrm{Fe}_{3} \mathrm{O}_{4}$, MIL, and GO. Due to their magnetic properties, the composites could also be recovered with an external magnetic field [30]. Pesticides dissolved in water bodies pose a serious threat to the aquatic as well as other life forms. Therefore, their removal is an important topic of research. In his direction, Liu et. al. synthesized a composite $\mathrm{Fe}_{3} \mathrm{O}_{4}$ GO-ß-CD/HKUST. This combination of metal oxide, copper-based MOF, and graphene oxide proved to be an excellent photocatalyst for the degradation of removal of ne onicotinoid insecticides, namely thiamethoxam imidacloprid acetamiprid, nitenpyram, dinotefuran, clothianidin, and thiacloprid. The removal was achieved from tap water which showed the practiced use of this composite [31]. Graphene-like carbon nitride $\left(\mathrm{g}-\mathrm{C}_{3} \mathrm{~N}_{4}\right)$ was used to prepare Z-scheme photocatalyst composite $\mathrm{Bi}_{2} \mathrm{O}_{3} / \mathrm{g}-\mathrm{C}_{3} \mathrm{~N}_{4}$ for studying its effectiveness in degrading phenol. The results showed it to be better than both $\mathrm{Bi}_{2} \mathrm{O}_{3}$ and $\mathrm{g}-\mathrm{C}_{3} \mathrm{~N}_{4}$ when used individually [32]. $\mathrm{Wu}$ and coworkers synthesized stable perovskite-type LaFe03/g-C3N4 quasi-polymeric heterostructure via the calcination method. The delayed electron-hole recombination was identified as the reason behind the increase in the bed lifetime of the charge carriers. Therefore, a Z-scheme charge carrier pathway ensued owing to the interaction at the $\mathrm{p}$-n heterojunction interface. This resulted in 16.9 times the greater photocatalytic activity of $\mathrm{LaFeO}_{3} / \mathrm{g}-\mathrm{C}_{3} \mathrm{~N}_{4}$ compared to pristine $\mathrm{g}-\mathrm{C}_{3} \mathrm{~N}_{4}$ in the degradation of the dye Brilliant Blue. Hydroxyl radicals, holes, and superoxide radicals were the primary reactive species in the process [33].

Jain and coworkers demonstrated heavy metal removal by metal oxide-carbon materials composites. The employed the co-precipitation method to synthesize the $\mathrm{ZnO} / \mathrm{AC}$ composites for the elimination of $\mathrm{Cd}$ (II), $\mathrm{Cu}(\mathrm{II})$, and $\mathrm{Cr}(\mathrm{Vl})$ species from tap water. Electrostatic attraction between the functional groups $-\mathrm{COOH}$ and $\mathrm{OH}$, present on the surface of adsorbent composites. The heavy metals were ascertained on the reason behind the removal of these metals [34]. Removal of Uranium is essential due to its radioactive nature and the occasional leakages that have happened over the years. To detect and remove Uranium from the aqueous sources, Guo et al. synthesized a magnetic ZIF-CD adsorbent. These $\mathrm{Fe}_{3} \mathrm{O}_{4}$-CMC@Z1F8@CD composites displayed better adsorption (173.60 $\mathrm{mg} / \mathrm{g}$ ) compared to the $\mathrm{Fe}_{3} \mathrm{O}_{4}$-CMC@Z1F (96.02 mg/g). The greater adsorption was attributed to the large surface area and abundant N/O containing functional groups present on the surface of the carbon dots [35].

Foul odor in the environment is a problem that needs immediate solutions. To achieve this goal, research has to been going on of developing e-noses to which can identify the malodorous substances. With an emphasis on longterm applications, metal oxide-based gas sensors (Figaro type) have been reported to be the most suitable [36]. Romain et al. worked for 3 years to establish the usefulness of tin oxide sensor arrays for identifying malodorous gases. Wastewater, paint shops, compost, and printing setups were found to be the sources that contributed mostly to the release of malodorous gases [37]. Humidity Charges were found to be crucial in the identification of different reducing gases by using $\mathrm{SnO}_{2}$ gas sensors. Therefore, the humidity calibration for e-noses equipped with $\mathrm{SnO}_{2}$ was prescribed to be time-dependent by Lonescu et al. to achieve desired improvements in the performance of these e-noses [38]. 


\section{Energy sustainability and solving carbon-related issues through metal oxide-based materials}

Energy conversion and storage are significant challenges faced by the modern world due to excessive industrialization. Therefore, low cost and high-efficiency catalysts are the need of the hour. In this context, metal oxides have been actively researched as viable options. Other applications of metal oxides in the energy sector include photoanodes for PEC Water splitting, intercalation cathodes for lithium-ion batteries, electrode materials for fuel cells, solar cells, supercapacitors, and electrochemical cells [39].

Simple metal oxides such as $\mathrm{TiO}_{2}, \mathrm{ZnO}_{2} \mathrm{WO}_{3}, \mathrm{BiVO}_{4}$, $\mathrm{Fe}_{2} \mathrm{O}_{3}$ have been found application as photoanodes for photoelectrochemical splitting of water, while $\mathrm{NiO}, \mathrm{Co}_{3} \mathrm{O}_{4}$, and $\mathrm{IrO}_{2}$ were reported to be efficient catalysts for water oxidation through electrocatalysis. The recent surge in nanotechnology has paved the way for various kinds of nanoparticles to develop sustainable and recyclable systems for energy conversion and storage. Dye-sensitized solar cells have been reported wherein $\mathrm{ZnO}$ and $\mathrm{TiO}_{2}$ nanoparticles have found use owing to their large surface area and electron transport abilities [40]. Doping has been one of the most favorite techniques of researchers worldwide. Both metal and non-metal dopants can be used to modulate the band gaps in the semiconductor metal oxides like
$\mathrm{ZnO}, \mathrm{TiO}_{2}$, $\mathrm{Ta}_{2} \mathrm{O}_{5}$, which makes it easier to access the visible light for carrying out photocatalysis [41,42].

Another Important strategy has been the introduction of intrinsic oxygen vacancies into the metal oxides. Methods have been explored to synthesize oxygen-deficient metal oxides to achieve PEC water splitting, usage in supercapacitors, solar cells, fuel cells, and LIBs. Such defects can modulate electronic and optical properties due to the rearrangement of inherent donor densities. The most common metal oxides that can be tuned in this manner are $\mathrm{Fe}_{2} \mathrm{O}_{3}, \mathrm{WO}_{3}, \mathrm{BiVO}_{4}$, and $\mathrm{TiO}_{2}$ [43]. Treatment of $\mathrm{TiO}_{2}$ nanowire arrays by hydrogen leads to oxygen vacancies during annealing at high temperatures. The reduction of $\mathrm{Ti}^{4+}$ to $\mathrm{Ti}^{3+}$ by hydrogen results in the formation of these vacancies since the overall charge is balanced. PEC performance of these oxygen vacancies containing $\mathrm{TiO}_{2}-\mathrm{NWs}$ coated electrodes was found to be significantly better than untreated $\mathrm{TiO}_{2}$-NWs [44]. Photoelectrode performance can be increased several folds by introducing oxygen defects that can synchronize with other complementary systems. One such report was published by Wang and coworkers in which they combined a microbial fuel cell with a PEC cell. Therefore, a self-sustained system for hydrogen gas liberation was obtained, which functioned continuously due to the $\mathrm{TiO}_{2}$ electrode that worked in the presence of solar light (Figure 2 [45]).

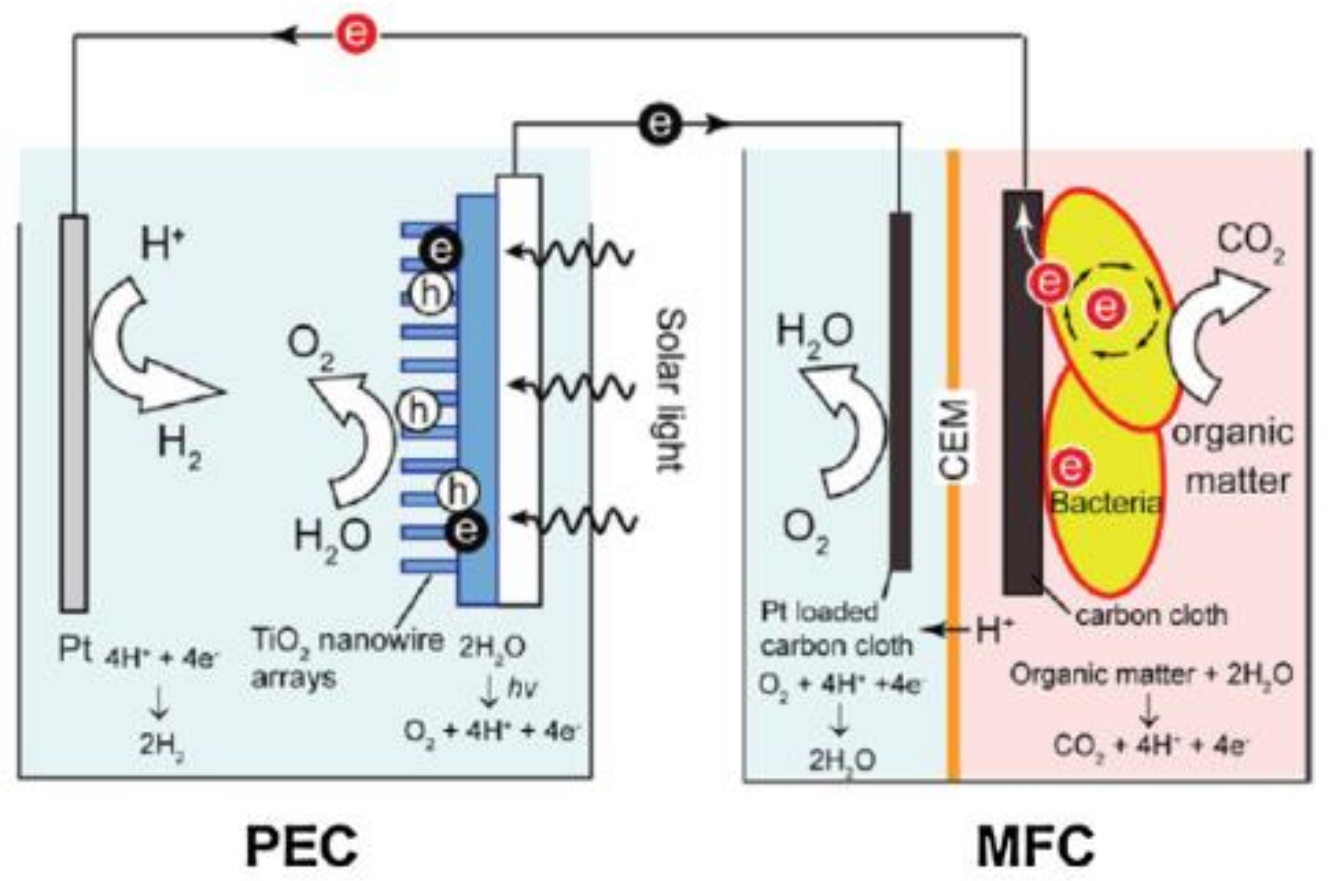

Figure 2. Schematic illustration of a PEC-MFC device [45].

$\mathrm{TiO}_{2}$ electrodes have been under investigation for increasing the efficiency of dye sensitized solar cells. $\mathrm{TiO}_{2}$ rutile NWs were found to improve the efficiency of DSSC from $0.28 \%$ to $0.45 \%$ for solar energy conversion. Hydrogen treatment of Degussa P25 resulted in oxygen vacancies that improved the solar energy conversion of DSSC to $13 \%$. Under natural sunlight, the enhancement was
9.30\%. The reason behind this improvement was ascertained to be the increase in electron density as a result of hydrogenation [46]. As a low-cost alternative to platinum, $\mathrm{WO}_{3}$ has been explored by Cheng et al. to operate as the counter electrode in DSSC. Since $\mathrm{I}_{3}{ }^{-}$gets reduced to $\mathrm{I}^{-}$during a complete cycle of electron transfer in DSSC, it is very important to have sites that can absorb $\mathrm{I}_{3}{ }^{-}$and reduce it to 
I-. The introduction of oxygen vacancies proved to be beneficial for this purpose. Thus, the use of $\mathrm{WO}_{3}$ and the introduction of oxygen vacancies therein resulted in a tremendous increase in conversion efficiency from $0.63 \%$ to $5.43 \%$ [47].

Supercapacitor efficiency can be significantly improved by oxygen defective metal oxides as demonstrated by Lu and coworkers, who used hydrogen treated $\mathrm{TiO}_{2}$ as a scaffold to support $\mathrm{MnO}_{2}$ nanoparticles, which act as pseudocapacitive materials. These nanoparticles were fabricated into $\mathrm{MnO}_{2}$ cores on top of which the $\mathrm{H}-\mathrm{TiO}_{2}$ were assembled. The as-prepared $\mathrm{H}_{-} \mathrm{TiO}_{2} @ \mathrm{MnO}_{2}$ core-shell NWs were used as a positive electrode in flexible solid-state asymmetric devices. With a slighter modification, $\mathrm{MnO}_{2}$ was replaced by carbon and the resulting $\mathrm{H}-\mathrm{TiO}_{2} @ \mathrm{C}$ core-shell NWs were used as the negative electrode. A high specific capacitance $(1.396 \mathrm{~F} / \mathrm{g})$ and a voltage window of $1.8 \mathrm{~V}$ was the characteristic feature of these supercapacitor devices [48]. Studies on the percentage of oxygen vacancies in $\mathrm{MoO}_{3}$ and the resulting influence on its capacitive performance revealed that the faster Kinetics of the oxygen-deficient $\mathrm{MoO}_{3}$ was directly related to the concentration of vacancies. Structural stability was also observed as a result of these defects. Therefore, repeated intercalation and de-intercalation of lithium was could be performed with very good cycling efficiency [49].

Liang and coworkers demonstrated application of oxygen-deficient Metal Oxide in lithium-ion batteries. They reduced. $\mathrm{TiO}_{2}$ with hydrogen and the resultant $\mathrm{TiO}_{2}$ inverse opal showed improvement in conductivity since the hydrogen-reduced $\mathrm{TiO}_{2}$ acted as an efficient current collector for $\mathrm{S}$ cathode. The oxygen vacancies enhanced S-Ti$\mathrm{O}$ bridges that acted as a medium for the sulfur-titanium oxide interaction while the $3 \mathrm{D}$ framework of $\mathrm{TiO}_{2}$ made the transport of Li ions and electrons easier. Therefore, the system achieved a high specific capacity of 1250 $\mathrm{mAh} / \mathrm{g}$. The oxygen deficiency was found to be the reason behind the excellent rate capability and reversible capacity due to the heightened electrical conductivity [50]. The zero/low emissions at ordinary temperatures and possessing high power densities make fuel cells promising sources of clean energy. Yet, costly catalysts, durability issues, and poor reliability hamper the commercialization of fuel cells. In this context, metal oxide-based materials have been found to solve the issue of catalysis to a large extent. For instance, upon annealing $\mathrm{MnO}_{2}$ in oxygen or argon atmosphere afforded nanocrystals of oxygen defective $\mathrm{MnO}_{2}$, which displayed ORR catalytic activity characterized by positive onset potential, low peroxide production, and greater current density. All of these parameters were found to be better than pure $\mathrm{MnO}_{2}$ because of the reduction in the kinetic barrier [51].

In a first of its kind, zero-dimensional CdS QDs were combined with $2 \mathrm{D} \mathrm{ZnO}$ nanosheets which resulted in the formation of heterostructures. In the presence of ultrasonic waves and $\mathrm{NaOH}$, a hydrothermal process was used to assemble a microflower-like structure for the 2D $\mathrm{ZnO}$ NSs upon which the CdS QDs were deposited by the process of successive ionic-layer absorption and reaction. The resultant $\mathrm{CdS} / \mathrm{ZnO}$ heterostructures were very effective in generating hydrogen by water splitting. A Z-scheme photocatalytic system that promoted electron-hole separation was at the core of the excellent catalytic ability of this heterostructure that was further enhanced by the fine-sized OD CdS and 2D ZnO and their intimate contact that diminished the migration resistance [52].

Energy storage is as important as energy generation. The batteries used traditionally lack the efficiency needed for the 21st century energy needs and cause severe pollution by leaking heavy metals and other toxic compounds into the environment. Therefore, other smart methods for storing energy, are being researched for quite some time. In the pursuit of preparing wearable energy storage devices, Lee et al. designed conductive textile with excellent toughness and high porosity via a two-step process wherein a metal oxide was coated onto the surface of cotton, followed by pyrolysis. The synthesized PCT$\mathrm{MOx} / \mathrm{ZnCl}_{2}$ composite textile $\left(\mathrm{MOx}=\mathrm{V}_{2} \mathrm{O}_{5}, \mathrm{Al}_{2} \mathrm{O}_{3}, \mathrm{ZnO}\right)$ were found to display unusual material toughness among with, the one prepared with V205 was the best. Introduction of $\mathrm{V} 205$ and $\mathrm{ZnCl}_{2}$ modified the graphene-like sheets in PCT to V-mediated cross-linked turbostatic carbon sheets and this was the reason behind the increase in material toughness. The PCT $-\mathrm{V}_{2} \mathrm{O}_{5} / \mathrm{ZnCl}_{2}$ was developed into a flexible solid-state supercapacitor using $\mathrm{PVA}-\mathrm{H}_{2} \mathrm{SO}_{4}$ as the gel electrolyte. The as-prepared supercapacitor was found to possess an energy density $\left(2.24 \mathrm{mWh} / \mathrm{cm}^{3}\right) 3$ times that of commercial supercapacitors, along with a power density $\left(585 \mathrm{~mW} / \mathrm{cm}^{3}\right)$ that is nearly twice as much as that of commercial Lithium ion batteries. This finding is expected to open up further research in a bid to develop wearable energy storage devices that can be commercialized at reasonable cost by using widely available metal oxides precursors [53].

Rapid climate change over the last century has led researchers as well as industries to think of new ways to remove the excess carbon accumulation in the atmosphere. In this attempt, several fields of research have emerged out among which, carbon dioxide photoreduction occupies a prominent place. The major criterion for any photocatalyst to work effectively in reducing $\mathrm{CO}_{2}$ lies in the extent of the gas it can adsorb. MOFs have been found suitable in this context since they possess Lewis acid sites to adsorb the $\mathrm{CO}_{2}$ molecules over the most abundant $\mathrm{N}_{2}$ gas molecules, from the atmosphere. MOFs can also house other sites with various functional groups and metals, to acts as centers for the catalytic activity [54]. The high adsorption capability of MOFs was combined with the favorable photocatalytic activity of $\mathrm{TiO}_{2}$ to produce $\mathrm{MOF} / \mathrm{TiO}_{2}$ systems for carbon dioxide reduction. By coating the nanocrystalline $\mathrm{TiO}_{2}$ onto the $\mathrm{Cu}_{3}(\mathrm{BTC})_{2}$ core, Xiong et al. developed as core-shell photocatalyst $\mathrm{Cu}_{3}(\mathrm{BTC})_{2} @ \mathrm{TiO}_{2}$ 
for selectively producing $\mathrm{CH}_{4}$ from $\mathrm{CO}_{2}$ with a 5 -fold enhancement over pure $\mathrm{TiO}_{2}$ [55]. Other examples of incorporating metal oxides into MOFs to achieve $\mathrm{CO}_{2}$ reduction have also been reported. These include $\mathrm{Zn}_{2} \mathrm{GeO}_{4} / \mathrm{Mg}$-MOF74, 0-ZnO/UiO-66- $\mathrm{NH}_{2}$, 0-ZnO/rGO/UiO-66- $\mathrm{NH}_{2}$, PCN$224(\mathrm{Cu}) / \mathrm{TiO}_{2}$, and QS- $\mathrm{Co}_{3} \mathrm{O}_{4} \mathrm{HoMSs}(\mathrm{ZIF}-67)$. Among these, the titanium and cobalt oxide based catalysts were reported to work under Xenon lamp, in water as the reaction medium, the $\mathrm{ZnO}$ based ones required the addition of acetonitrile or $\mathrm{NaHCO}_{3}$ to the reaction medium for their activity [56].

Conversion of $\mathrm{CO}_{2}$ into useful fuels and other valuable precursors for industries is a great way to reduce the carbon footprint and cut the cost of production in a single shot. Therefore, carbon dioxide to methanol conversion could be economically viable if low-cost catalytic systems can be developed. In this context, Senanayake and coworkers reported the synthesis of $\mathrm{CeO}_{\mathrm{x}} / \mathrm{Cu}(111)$ and $\mathrm{ZnO} / \mathrm{Cu}(111)$ catalysts for the easy conversion of $\mathrm{CO}_{2}$ to methanol via stabilization of the intermediate $\mathrm{CO}_{2}{ }^{\delta-}$ species. The role of metal oxide was especially investigated, and they found that the deposition of $\mathrm{CeO}_{\mathrm{x}}$ and $\mathrm{ZnO}$ NPs on the $\mathrm{Cu}(111)$ surface decreases the activation energy for the methanol synthesis from $25 \mathrm{kcal} / \mathrm{mol}$ on pristine $\mathrm{Cu}(111)$ to 13 and $16 \mathrm{kcal} / \mathrm{mol}$ on $\mathrm{CeO}_{\mathrm{x}} / \mathrm{Cu}(111)$ and $\mathrm{ZnO} / \mathrm{Cu}(111)$, respectively. Moreover, strong metal-oxide interactions were observed as a result of the inverse oxide/metal configuration. $\mathrm{Ce}^{3+}$ was established as the site for stabilizing the intermediate $\mathrm{CO}_{2}{ }^{\delta-}$ species. Overall, the metal-metal oxide interfaces, $\mathrm{CeO}_{\mathrm{x}}-\mathrm{Cu}$ and $\mathrm{ZnO}-\mathrm{Cu}$, were considered critical in the hydrogenation of $\mathrm{CO}_{2}$ [57].

Use of oxygen-deficient metal oxides for reducing $\mathrm{CO}_{2}$ into useful chemicals has been demonstrated by Gao et al. in their report about the partially oxidized atomic cobalt layer that displayed excellent selectivity (90\%) for formate production. With an overpotential of $0.24 \mathrm{~V}$, this metal oxide system is an outstanding one in terms of $\mathrm{CO}_{2}$ reduction catalysis. The difficulty in ascertaining the possible mechanism was due to the difficulty in identifying active sites, which in turn, was because of the mixture of cobalt oxide and metallic cobalt particles in the system [58]. However, such systems can provide further insights and pave the way for designing better photocatalysts if the role of oxygen deficiency present therein, can be investigated.

Interfacial charge transfer was observed in the case of $\mathrm{LaFeO}_{3}-\mathrm{TiO}_{2}$ nanocomposites prepared by Humayun et. al. Increased specific surface area, and improved harvesting of visible light, in addition to N-doping were also considered to be causes behind the efficiency shown by this system (Figure 3) [59].

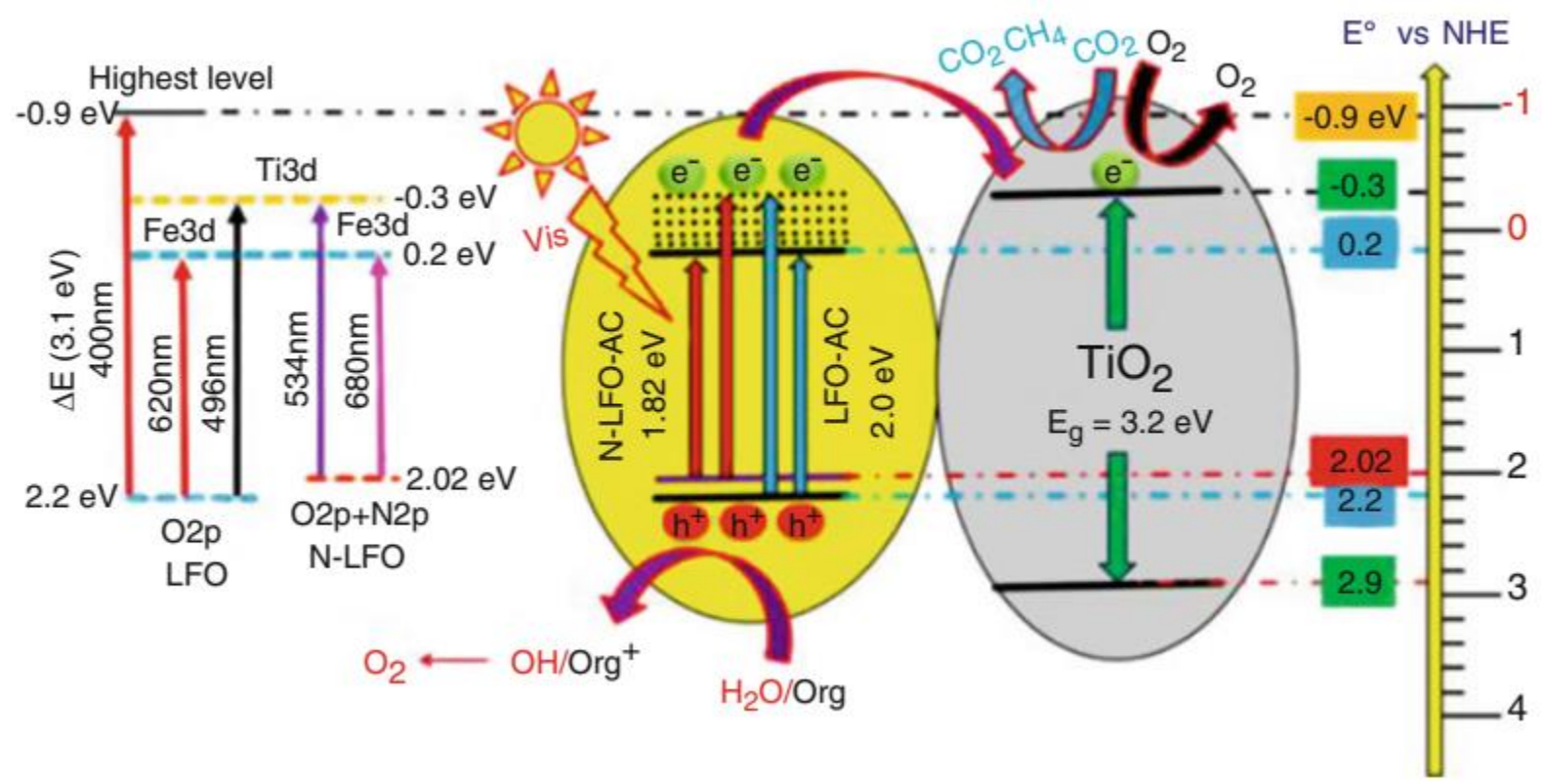

Figure 3. Interfacial charge transfer in $\mathrm{LaFeO}_{3}-\mathrm{TiO}_{2}$ nanocomposites catalyzed photoreduction of $\mathrm{CO}_{2}[59]$.

A heterojunction photocatalyst g- $\mathrm{C}_{3} \mathrm{~N}_{4} / \mathrm{NaNbO}_{3}$ also displayed good photocatalytic carbon dioxide reduction wherein the carbon nitride provided the light harvesting ability while the metal oxide provided the necessary bandgap for the process. An artificial photosynthetic system reported by Zhou et al. was found to have mimicked the real leaves as a result of its 3D structural features. Sunlight as the energy source, carbon dioxide as the carbon source and water as the electron donor was achieved through this 3D APS architecture. $\mathrm{SrTiO}_{3}$ was identified as the active site for breakdown of $\mathrm{CO}_{2}$, and cocatalysts like $\mathrm{Pt}, \mathrm{Ag}, \mathrm{Au}$, and $\mathrm{Cu}$ were found to be suitable for loading. Interestingly, this system was found to have morphological similarities with natural leaf. Therefore, construction of such systems is quite promising in neutralizing the excess carbon content in the environment. 


\section{Conclusion}

Metal-oxide-based materials that demonstrate various properties such as electrical, magnetic, mechanical, optical, catalytic, etc., gained significant importance in various parts of industrial applications. Metal-organic frameworks (MOFs) consist of metallic ions and organic ligands, which have gained substantial interest due to their techno-economic advantages with relatively low cost of metal oxide semiconductors than pure metallic nanoparticles that can provide great alternative photocatalysts especially considering the adjustable bandgaps and synergistic effects. Also, the oxide-MOFs-based compositions have high porosity high surface area with adjustable pore size from the features perspective. Recently MOFs and carbon-based metal oxides are considered for a wide range of applications. Specifically, their application for energy suitability can be counted for hydrogen energy and battery applications for storage catalysts systems, fuel and solar cells, sensors, pyroelectric, etc., applications at industry scale, and more importantly, supercapacitors applications. Therefore, this study reviews the subject to develop essential information that the subject will pursue in the next phase in details of frameworking, planning, modeling, design, and application of metal-oxide-based materials in energy environmental sustainability.

\section{References}

[1] Emam HE, Ahmed HB, Gomaa E, Helal MH, Abdelhameed RM (2019) "Doping of silver vanadate and silver tungstate nanoparticles for enhancement the photocatalytic activity of MIL-125-NH2 in dye degradation" Journal of Photochemistry and Photobiology A: Chemistry (vol. 383, pp. 111986) https://doi.org/10.1016/j.jphotochem.2019.111986

[2] Zhang C, Ai L, Jiang J (2015) “Graphene hybridized photoactive iron terephthalate with enhanced photocatalytic activity for the degradation of rhodamine $b$ under visible light" Ind Eng Chem Res (vol. 54, no. 1, pp. 153-163) https://doi.org/10.1021/ie504111y

[3] Rad M, Dehghanpour S (2016) "ZnO as an efficient nucleating agent and morphology template for rapid, facile and scalable synthesis of MOF-46 and ZnO@MOF-46 with selective sensing properties and enhanced photocatalytic ability" RSC Adv (vol. 6, no. 66, pp. 61784-61793) https://doi.org/10.1039/C6RA12410K

[4] Wang X, Liu J, Leong S, Lin X, Wei J, et al. (2016) "Rapid construction of ZnO@ZIF-8 heterostructures with size-selective photocatalysis properties" ACS Appl Mater Interfaces (vol. 8, no. 14, pp. 9080-9087) https://doi.org/10.1021/acsami.6b00028

[5] Mahmoodi NM, Taghizadeh A, Taghizadeh M, Abdi J (2019) "In situ deposition of $\mathrm{Ag} / \mathrm{AgCl}$ on the surface of magnetic metal-organic framework nanocomposite and its application for the visible-light photocatalytic degradation of Rhodamine dye" Journal of Hazardous Materials (vol. 378, pp. 120741) https://doi.org/10.1016/j.jhazmat.2019.06.018

[6] Jiang D, Xu P, Wang H, Zeng G, Huang D, et al. (2018) "Strategies to improve metal organic frameworks photocatalyst's performance for degradation of organic pollutants" Coordination Chemistry Reviews (vol. 376, pp. 449-466) https://doi.org/10.1016/j.ccr.2018.08.005

[7] Xie M-H, Shao R, Xi X-G, Hou G-H, Guan R-F, et al. (2017) "Metal-organic framework photosensitized TiO2 co-catalyst: A facile strategy to achieve a high efficiency photocatalytic system" Chemistry - A European Journal (vol. 23, no. 16, pp. 3931-3937) https://doi.org/10.1002/chem.201605282

[8] Li H, Li Q, He Y, Zhang N, Xu Z, et al. (2018) "Facile fabrication of magnetic metal-organic framework composites for the highly selective removal of cationic dyes" Materials (vol. 11, no. 5, pp. 744) https://doi.org/10.3390/ma11050744

[9] Zhao X, Liu S, Tang Z, Niu H, Cai Y, et al. (2015) "Synthesis of magnetic metal-organic framework (MOF) for efficient removal of organic dyes from water" Sci Rep (vol. 5, no. 1, pp. 11849) https://doi.org/10.1038/srep11849

[10] Zhang M, Qiao R, Hu J (2020) "Engineering Metal-Organic Frameworks (MOFs) for Controlled Delivery of Physiological Gaseous Transmitters" Nanomaterials (vol. 10, no. 6, pp. 1134) https://doi.org/10.3390/nano10061134

[11] Li Y, Zhou X, Dong L, Lai Y, Li S, et al. (2019) "Magnetic metal-organic frameworks nanocomposites for negligibledepletion solid-phase extraction of freely dissolved polyaromatic hydrocarbons" Environmental Pollution (vol. 252, pp. 1574-1581) https://doi.org/10.1016/j.envpol.2019.04.137

[12] Torretta V, Katsoyiannis IA, Viotti P, Rada EC (2018) “Critical review of the effects of glyphosate exposure to the environment and humans through the food supply chain" Sustainability (vol. 10, no. 4, pp. 950) https://doi.org/10.3390/su10040950

[13] Danish MSS, Bhattacharya A, Stepanova D, Mikhaylov A, Grilli ML, et al. (2020) "A systematic review of metal oxide applications for energy and environmental sustainability" Metals (vol. 10, no. 12, pp. 1604) https://doi.org/10.3390/met10121604

[14] Danish MSS, Estrella LL, Alemaida IMA, Lisin A, Moiseev N, et al. (2021) "Photocatalytic applications of metal oxides for sustainable environmental remediation" Metals (vol. 11, no. 1, pp. 80) https://doi.org/10.3390/met11010080

[15] He X, Nguyen V, Jiang Z, Wang D, Zhu Z, et al. (2018) "Highly-oriented one-dimensional MOF-semiconductor nanoarrays for efficient photodegradation of antibiotics" Catal Sci Technol (vol. 8, no. 8, pp. 2117-2123) https://doi.org/10.1039/C8CY00229K

[16] Moradi SE, Haji Shabani AM, Dadfarnia S, Emami S (2016) "Effective removal of ciprofloxacin from aqueous solutions using magnetic metal-organic framework sorbents: mechanisms, isotherms and kinetics" J IRAN CHEM SOC (vol. 13, no. 9, pp. 1617-1627) https://doi.org/10.1007/s13738016-0878-y

[17] Huo J-B, Xu L, Chen X, Zhang Y, Yang J-CE, et al. (2019) “Direct epitaxial synthesis of magnetic Fe304@Ui0-66 composite for efficient removal of arsenate from water" $M i$ croporous and Mesoporous Materials (vol. 276, pp. 68-75) https://doi.org/10.1016/j.micromeso.2018.09.017 
[18] Ma Y, Xu G, Wei F, Cen Y, Xu X, et al. (2018) “One-pot synthesis of a magnetic, ratiometric fluorescent nanoprobe by encapsulating $\mathrm{Fe} 304$ magnetic nanoparticles and dualemissive rhodamine $\mathrm{b}$ modified carbon dots in metal-organic framework for enhanced $\mathrm{HClO}$ sensing" ACS Appl Mater Interfaces (vol. 10, no. 24, pp. 20801-20805) https://doi.org/10.1021/acsami.8b05643

[19] Gu C, Xiong S, Zhong Z, Wang Y, Xing W (2017) "A promising carbon fiber-based photocatalyst with hierarchical structure for dye degradation" RSC Adv (vol. 7, no. 36, pp. 22234-22242) https://doi.org/10.1039/C7RA02583A

[20] Nekouei S, Nekouei F, Kargarzadeh H (2018) "Synthesis of $\mathrm{ZnO}$ photocatalyst modified with activated carbon for a perfect degradation of ciprofloxacin and its secondary pollutants" Applied Organometallic Chemistry (vol. 32, no. 3, pp. e4198) https://doi.org/10.1002/aoc.4198

[21] Atchudan R, Edison TNJI, Perumal S, Karthik N, Karthikeyan D, et al. (2018) "Concurrent synthesis of nitrogen-doped carbon dots for cell imaging and ZnO@nitrogen-doped carbon sheets for photocatalytic degradation of methylene blue" Journal of Photochemistry and Photobiology A: Chemistry (vol. 350, pp. 75-85) https://doi.org/10.1016/j.jphotochem.2017.09.038

[22] Wang F, Zhou Y, Pan X, Lu B, Huang J, et al. (2018) "Enhanced photocatalytic properties of $\mathrm{ZnO}$ nanorods by electrostatic self-assembly with reduced graphene oxide" Phys Chem Chem Phys (vol. 20, no. 10, pp. 6959-6969) https://doi.org/10.1039/C7CP06909J

[23] Jo W-K, Kumar S, Isaacs MarkA, Lee AF, Karthikeyan S (2017) "Cobalt promoted $\mathrm{TiO} 2 / \mathrm{GO}$ for the photocatalytic degradation of oxytetracycline and Congo Red" Applied Catalysis B: Environmental (vol. 201, pp. 159-168) https://doi.org/10.1016/j.apcatb.2016.08.022

[24] Ahmed B, Ojha AK, Singh A, Hirsch F, Fischer I, et al. (2018) "Well-controlled in-situ growth of 2D WO3 rectangular sheets on reduced graphene oxide with strong photocatalytic and antibacterial properties" Journal of Hazardous Materials (vol. 347, pp. 266-278) https://doi.org/10.1016/j.jhazmat.2017.12.069

[25] Gan L, Xu L, Shang S, Zhou X, Meng L (2016) "Visible light induced methylene blue dye degradation photo-catalyzed by W03/graphene nanocomposites and the mechanism" Ceramics International (vol. 42, no. 14, pp. 15235-15241) https://doi.org/10.1016/j.ceramint.2016.06.160

[26] Taha AA, Li F (2014) "Porous WO3-carbon nanofibers: high-performance and recyclable visible light photocatalysis" Catal Sci Technol (vol. 4, no. 10, pp. 3601-3605) https://doi.org/10.1039/C4CY00777H

[27] Song B, Wang T, Sun H, Shao Q, Zhao J, et al. (2017) "Twostep hydrothermally synthesized carbon nanodots/WO3 photocatalysts with enhanced photocatalytic performance" Dalton Trans (vol. 46, no. 45, pp. 15769-15777) https://doi.org/10.1039/C7DT03003G

[28] Jeevitha G, Abhinayaa R, Mangalaraj D, Ponpandian N (2018) "Tungsten oxide-graphene oxide (WO3-GO) nanocomposite as an efficient photocatalyst, antibacterial and anticancer agent" Journal of Physics and Chemistry of Solids (vol. 116, pp. 137-147) https://doi.org/10.1016/j.jpcs.2018.01.021

[29] Lee C-G, Javed H, Zhang D, Kim J-H, Westerhoff P, et al. (2018) "Porous electrospun fibers embedding $\mathrm{TiO} 2$ for adsorption and photocatalytic degradation of water pollutants" Environ Sci Technol (vol. 52, no. 7, pp. 4285-4293) https://doi.org/10.1021/acs.est.7b06508

[30] Gong Q, Liu Y, Dang Z (2019) "Core-shell structured Fe304@G0@MIL-100(Fe) magnetic nanoparticles as heterogeneous photo-Fenton catalyst for 2,4-dichlorophenol degradation under visible light" J Hazard Mater (vol. 371, pp. 677-686) https://doi.org/10.1016/j.jhazmat.2019.03.019

[31] Liu G, Li L, Xu D, Huang X, Xu X, et al. (2017) “Metal-organic framework preparation using magnetic graphene oxide- $\beta$ cyclodextrin for neonicotinoid pesticide adsorption and removal" Carbohydrate Polymers (vol. 175, pp. 584-591) https://doi.org/10.1016/j.carbpol.2017.06.074

[32] He R, Zhou J, Fu H, Zhang S, Jiang C (2018) “Room-temperature in situ fabrication of Bi203/g-C3N4 direct Z-scheme photocatalyst with enhanced photocatalytic activity" $A p$ plied Surface Science (vol. 430, pp. 273-282) https://doi.org/10.1016/j.apsusc.2017.07.191

[33] Wu Y, Wang H, Tu W, Liu Y, Tan YZ, et al. (2018) "Quasipolymeric construction of stable perovskite-type LaFe03/g-C3N4 heterostructured photocatalyst for improved Z-scheme photocatalytic activity via solid p-n heterojunction interfacial effect" Journal of Hazardous Materials (vol. 347, pp. 412-422) https://doi.org/10.1016/j.jhazmat.2018.01.025

[34] Jain M, Yadav M, Kohout T, Lahtinen M, Garg VK, et al. (2018) "Development of iron oxide/activated carbon nanoparticle composite for the removal of $\mathrm{Cr}(\mathrm{VI}), \mathrm{Cu}(\mathrm{II})$ and $\mathrm{Cd}(\mathrm{II})$ ions from aqueous solution" Water Resources and Industry (vol. 20, pp. 54-74) https://doi.org/10.1016/j.wri.2018.10.001

[35] Guo X, Liu Q, Liu J, Zhang H, Yu J, et al. (2019) "Magnetic metal-organic frameworks/carbon dots as a multifunctional platform for detection and removal of uranium" $A p$ plied Surface Science (vol. 491, pp. 640-649) https://doi.org/10.1016/j.apsusc.2019.06.108

[36] Romain AC, Nicolas J (2010) "Long term stability of metal oxide-based gas sensors for e-nose environmental applications: An overview" Sensors and Actuators B: Chemical (vol. 146, no. 2, pp. 502-506) https://doi.org/10.1016/j.snb.2009.12.027

[37] Romain A-C, André Ph, Nicolas J (2002) "Three years experiment with the same tin oxide sensor arrays for the identification of malodorous sources in the environment" Sensors and Actuators B: Chemical (vol. 84, no. 2, pp. 271277) https://doi.org/10.1016/S0925-4005(02)00036-9

[38] Ionescu R, Vancu A, Tomescu A (2000) “Time-dependent humidity calibration for drift corrections in electronic noses equipped with $\mathrm{SnO} 2$ gas sensors" Sensors and Actuators B: Chemical (vol. 69, no. 3, pp. 283-286) https://doi.org/10.1016/S0925-4005(00)00508-6

[39] Wang G, Yang Y, Han D, Li Y (2017) “Oxygen defective metal oxides for energy conversion and storage" Nano Today (vol. 13 , pp. 23-39) https://doi.org/10.1016/j.nantod.2017.02.009

[40] O’Regan B, Grätzel M (1991) “A low-cost, high-efficiency solar cell based on dye-sensitized colloidal TiO 2 films" $\mathrm{Na}$ ture (vol. 353, no. 6346, pp. 737-740) https://doi.org/10.1038/353737a0 
[41] Yang X, Wolcott A, Wang G, Sobo A, Fitzmorris RC, et al. (2009) "Nitrogen-doped $\mathrm{ZnO}$ nanowire arrays for photoelectrochemical water splitting" Nano Lett (vol. 9, no. 6, pp. 2331-2336) https://doi.org/10.1021/nl900772q

[42] Hoang S, Guo S, Hahn NT, Bard AJ, Mullins CB (2012) "Visible light driven photoelectrochemical water oxidation on nitrogen-modified TiO2 nanowires" Nano Lett (vol. 12, no. 1, pp. 26-32) https://doi.org/10.1021/nl2028188

[43] Kenney MJ, Gong M, Li Y, Wu JZ, Feng J, et al. (2013) “Highperformance silicon photoanodes passivated with ultrathin nickel films for water oxidation" Science (vol. 342, no. 6160, pp. 836-840) https://doi.org/10.1126/science.1241327

[44] Wang G, Wang H, Ling Y, Tang Y, Yang X, et al. (2011) "Hydrogen-treated $\mathrm{TiO} 2$ nanowire arrays for photoelectrochemical water splitting" Nano Lett (vol. 11, no. 7, pp. 3026-3033) https://doi.org/10.1021/nl201766h

[45] Wang H, Qian F, Wang G, Jiao Y, He Z, et al. (2013) "Selfbiased solar-microbial device for sustainable hydrogen generation" ACS Nano (vol. 7, no. 10, pp. 8728-8735) https://doi.org/10.1021/nn403082m

[46] Yang Y, Ling Y, Wang G, Liu T, Wang F, et al. (2015) "Photohole induced corrosion of titanium dioxide: Mechanism and solutions" Nano Lett (vol. 15, no. 10, pp. 7051-7057) https://doi.org/10.1021/acs.nanolett.5b03114

[47] Cheng L, Hou Y, Zhang B, Yang S, Guo JW, et al. (2013) "Hydrogen-treated commercial WO3 as an efficient electrocatalyst for triiodide reduction in dye-sensitized solar cells" Chem Commun (vol. 49, no. 53, pp. 5945-5947) https://doi.org/10.1039/C3CC42206B

[48] Lu X, Yu M, Wang G, Zhai T, Xie S, et al. (2013) "HTiO2@Mn02//H-TiO2@C core-shell nanowires for high prformance and flexible asymmetric supercapacitors" Advanced Materials (vol. 25, no. 2, pp. 267-272) https://doi.org/10.1002/adma.201203410

[49] Kang Q, Cao J, Zhang Y, Liu L, Xu H, et al. (2013) "Reduced Ti02 nanotube arrays for photoelectrochemical water splitting" J Mater Chem A (vol. 1, no. 18, pp. 5766-5774) https://doi.org/10.1039/C3TA10689F

[50] Liang Z, Zheng G, Li W, Seh ZW, Yao H, et al. (2014) "Sulfur cathodes with hydrogen reduced titanium dioxide inverse opal structure" ACS Nano (vol. 8, no. 5, pp. 5249-5256) https://doi.org/10.1021/nn501308m

[51] Tan H, Zhao Z, Niu M, Mao C, Cao D, et al. (2014) “A facile and versatile method for preparation of colored TiO2 with enhanced solar-driven photocatalytic activity" Nanoscale (vol. 6, no. 17, pp. 10216-10223) https://doi.org/10.1039/C4NR02677B

[52] Ma D, Shi J-W, Zou Y, Fan Z, Ji X, et al. (2017) "Highly efficient photocatalyst based on a CdS quantum Dots/ZnO nanosheets $0 \mathrm{D} / 2 \mathrm{D}$ heterojunction for hydrogen evolution from water splitting" ACS Appl Mater Interfaces (vol. 9, no. 30, pp. 25377-25386) https://doi.org/10.1021/acsami.7b08407

[53] Lam DV, Won S, Shim HC, Kim J-H, Lee S-M (2019) “Turning cotton into tough energy textile via metal oxide assisted carbonization" Carbon (vol. 153, pp. 257-264) https://doi.org/10.1016/j.carbon.2019.07.010

[54] Younis SA, Kwon EE, Qasim M, Kim K-H, Kim T, et al. (2020) "Metal-organic framework as a photocatalyst: Progress in modulation strategies and environmental/energy applications" Progress in Energy and Combustion Science (vol. 81, pp. 100870) https://doi.org/10.1016/j.pecs.2020.100870

[55] Li R, Wu S, Wan X, Xu H, Xiong Y (2016) “Cu/TiO2 octahedral-shell photocatalysts derived from metal-organic framework@semiconductor hybrid structures" Inorg Chem Front (vol. 3, no. 1, pp. 104-110) https://doi.org/10.1039/C5QI00205B

[56] Kidanemariam A, Lee J, Park J (2019) "Recent innovation of metal-organic frameworks for carbon dioxide photocatalytic reduction" Polymers (vol. 11, no. 12, pp. 2090) https://doi.org/10.3390/polym11122090

[57] Senanayake SD, Ramírez PJ, Waluyo I, Kundu S, Mudiyanselage K, et al. (2016) "Hydrogenation of $\mathrm{CO} 2$ to methanol on $\mathrm{CeOx} / \mathrm{Cu}(111)$ and $\mathrm{ZnO} / \mathrm{Cu}(111)$ catalysts: Role of the metal-oxide interface and importance of Ce3+ site" J Phys Chem $C$ (vol. 120, no. 3, pp. 1778-1784) https://doi.org/10.1021/acs.jpcc.5b12012

[58] Gao S, Lin Y, Jiao X, Sun Y, Luo Q, et al. (2016) "Partially oxidized atomic cobalt layers for carbon dioxide electroreduction to liquid fuel" Nature (vol. 529, no. 7584, pp. 68-71) https://doi.org/10.1038/nature16455

[59] Humayun M, Qu Y, Raziq F, Yan R, Li Z, et al. (2016) "Exceptional visible-light activities of TiO2-coupled N-doped porous perovskite $\mathrm{LaFeO} 3$ for 2,4-dichlorophenol decomposition and CO2 conversion" Environ Sci Technol (vol. 50, no. 24, pp. 13600-13610) https://doi.org/10.1021/acs.est.6b04958 